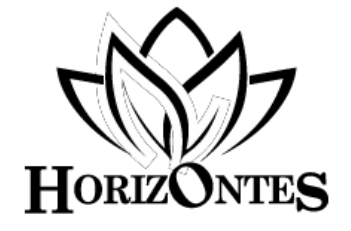

www.revistahorizontes.org
Horizontes. Revista de Investigación en Ciencias de la Educación Https://doi.org/10.33996/revistahorizontes.v4i16.131

Octubre - Diciembre 2020

Volumen 4 / No. 16

ISSN: $2616-7964$

ISSN-L: $2616-7964$

pp. $483-498$

\title{
Diseño de textos digitales accesibles para estudiantes con discapacidad visual
}

\author{
Design of accessible digital texts for students with visual disabilities \\ Projeto de textos digitais acessíveis para alunos com deficiência visual
}

ARTÍCULO DE INVESTIGACIÓN

Karina Maribel Castillo Pinos
karina.castillopinos1414@gmail.com
ORCID: 0000-0002-1154-1297

Miguel Eduardo Vásconez Campos

miguelvasconez_campos@hotmail.com

ORCID: 0000-0002-6644-3918

\author{
Alcívar Alejandro Vega Sánchez \\ alejovs28@gmail.com \\ ORCID: 0000-0002-4925-6360
}

María Eugenia Ochoa Guerrero

marules85@hotmail.com

ORCID: 0000-0002-3866-8781

\section{Universidad Nacional de Educación, Ecuador}

Recibido 10 de noviembre 2020 | Arbitrado y aceptado 27 de noviembre 2020 | Publicado en diciembre 2020

\section{RESUMEN}

El presente artículo muestra una propuesta de un diseño de textos digitales accesibles para estudiantes con discapacidad visual de la Unidad de Educación Especial Claudio Neira Garzón en Ecuador. Así mismo, se realiza una revisión teórica en torno a la educación inclusiva, acerca de la discapacidad visual y textos digitales accesibles, considerando las necesidades educativas de los estudiantes con discapacidad visual. Se trabajó con un enfoque cualitativo, bajo un proceso de análisis de desarrollo sistémico, aplicando la metodología de la investigación acción participativa, donde se muestra una propuesta que tiene como finalidad transformar la realidad en el ámbito educativo del colectivo con esta condición. Los resultados obtenidos en la aplicación de la encuesta a los docentes la institución educativa, permitieron concluir que los mismos, carecen de los conocimientos básicos para elaborar textos digitales accesibles para estudiantes con discapacidad visual, por lo cual se elaboró una propuesta para mejorar este aspecto.

Palabras clave: Discapacidad visual; recursos digitales accesibles; accesibilidad, educación inclusiva

\section{ABSTRACT}

This article shows a proposal for a design of accessible digital texts for students with visual disabilities of the Claudio Neira Garzón Special Education Unit in Ecuador. Likewise, a theoretical review is carried out around inclusive education, about visual disability and accessible digital texts, considering the educational needs of students with visual disabilities. We worked with a qualitative approach, under a systemic development analysis process, applying the participatory action research methodology, where a proposal is shown that aims to transform reality in the educational field of the group with this condition. The results obtained in the application of the survey to the teachers of the educational institution, allowed to conclude that they lack the basic knowledge to elaborate accessible digital texts for students with visual disabilities, for which a proposal was elaborated to improve this aspect.

Key words Visual impairment; accessible digital resources; accessibility, inclusive education 


\section{RESUMO}

Este artigo apresenta uma proposta de desenho de textos digitais acessíveis para alunos com deficiência visual da Unidade de Educação Especial Claudio Neira Garzón, no Equador. Da mesma forma, é realizada uma revisão teórica em torno da educação inclusiva, sobre deficiência visual e textos digitais acessíveis, considerando as necessidades educacionais dos alunos com deficiência visual. Trabalhamos com uma abordagem qualitativa, sob um processo de análise do desenvolvimento sistêmico, aplicando a metodologia da pesquisa-ação participativa, onde se apresenta uma proposta que visa transformar a realidade no campo educacional do grupo com essa condição. Os resultados obtidos na aplicação da pesquisa aos professores da instituição de ensino, permitiram concluir que estes carecem de conhecimentos básicos para a elaboração de textos digitais acessíveis para alunos com deficiência visual, para os quais foi elaborada uma proposta para melhorar este aspecto.

Palavras-chave: Deficiência visual; recursos digitais acessíveis; acessibilidade, educação inclusiva

\section{INTRODUCCIÓN}

En la actualidad los avances científicos, sociales, económicos, educativos, etc., se han caracterizado por la incorporación de la tecnología, es así que hoy en día hablamos de una sociedad de la información, haciendo hincapié en la importante e innegable influencia de la tecnología en esta metamorfosis educativa que estamos experimentando.

El conjunto de transformaciones sociales propiciadas por la innovación tecnológica y por el desarrollo de las tecnologías de la información y de la comunicación, los cambios en las relaciones sociales y una nueva concepción de las relaciones tecnologíasociedad que condiciona la posición de las tecnologías frente a la educación, están provocando que el sistema educativo, una de las instituciones sociales por excelencia, se encuentre inmerso en un proceso de cambios (Salinas, 2014).

Por lo tanto, la educación de nuestro país, y a nivel mundial, se encuentra frente a un doble reto; ya que por un lado debe formar seres humanos preparados para responder a una sociedad cada vez más digitalizada, y por otro lado, cumplir con los principios de la inclusión, lo cual amerita brindar recursos accesibles para la diversidad de estudiantes que encontramos en nuestras aulas. En este sentido, resulta necesario indicar que la diversidad es un término amplio y específico a la vez. Amplio ya que abarca las diferencias inherentes al ser humano, englobando características culturales, religiosas, económicas, sexuales, de condición física, sensorial o intelectual. Sin embargo, la diversidad es específica en tanto se debe considerar las particularidades de aprendizaje, en el ámbito educativo, que a su vez se convierten en indicadores para determinar los apoyos que debe generar el entorno. Por lo antes mencionado, en esta investigación nos enfocaremos en aquellos recursos digitales que requieren los educandos que presentan discapacidad visual.

Como es sabido, los responsables de brindar recursos didácticos inclusivos son los docentes, por lo que el objetivo general de esta investigación, consiste en proponer el diseño de textos digitales accesibles para estudiantes con discapacidad visual de la Unidad de Educación Especial "Claudio Neira Garzón", a través de ciertas pautas y herramientas, con la finalidad de mejorar el proceso educativo de estudiantes que presentan esta condición, así mismo se establecieron los siguientes objetivos específicos: 1) fundamentar teóricamente los aspectos relacionados con la discapacidad visual y el uso de recursos digitales accesibles, 2) Determinar el nivel de conocimientos que presentan los docentes de la Unidad de Educación Especial "Claudio Neira Garzón" sobre el diseño de textos digitales accesibles para estudiantes con discapacidad visual y 3) 
Elaborar y validar una propuesta para diseñar textos digitales accesibles para estudiantes con esta limitación.

En base a lo anteriormente mencionado sobre la importancia del uso de recursos digitales en la educación y centrándonos en los objetivos planteados para el presente trabajo, surge la siguiente pregunta de investigación: ¿Cómo diseñar textos digitales accesibles para estudiantes con discapacidad visual?, inquietud que intentaremos responder a lo largo del desarrollo de esta propuesta.

\section{MÉTODO}

La metodología para el análisis de la información fue bajo el enfoque cualitativo puesto que se desarrolló un proceso sistémico riguroso orientado en la toma de decisiones, en este caso al realizar la propuesta sobre diseño de textos digitales accesibles para estudiantes con discapacidad visual, es decir, que se toman acciones de forma directa para intervenir en la problemática que se está observando o investigando (Albert, 2007).

Así mismo, se ha optado por desarrollar la investigación acción (IA) modelo que busca el cambio en el desarrollo participativo respondiendo a las dinámicas sociales, en este sentido la IA tomó su lugar como un proceso metodológico que fue acorde a las necesidades de una población estudiada, con la intención de obtener un sentido más flexible respecto a la toma de decisiones. El valor fundamental de la misma se basa en el compartir de ideas, cuestionamientos y problemas que puedan existir, para poder dar respuesta a los mismos, y de esa manera lograr transformar la realidad social.

\section{RESULTADOS Y DISCUSIÓN}

Los datos que se presentan a continuación, son el resultado de la aplicación de la encuesta dirigida a los docentes de la Unidad de Educación Especial Claudio Neira Garzón, en relación al nivel de conocimientos con los que cuenta el personal docente sobre el diseño de textos digitales accesibles para estudiantes con discapacidad visual. Cabe destacar que este cuestionario estaba estructurado por 8 preguntas sobre la temática antes mencionada, seguidamente se expone la información que se obtuvo.

Con relación a la pregunta número 1 en el gráfico 1 destaca las impresiones recogidas de la población encuestada sobre los recursos tecnológicos que favorecen el proceso de enseñanza y aprendizaje de los estudiantes con discapacidad visual.
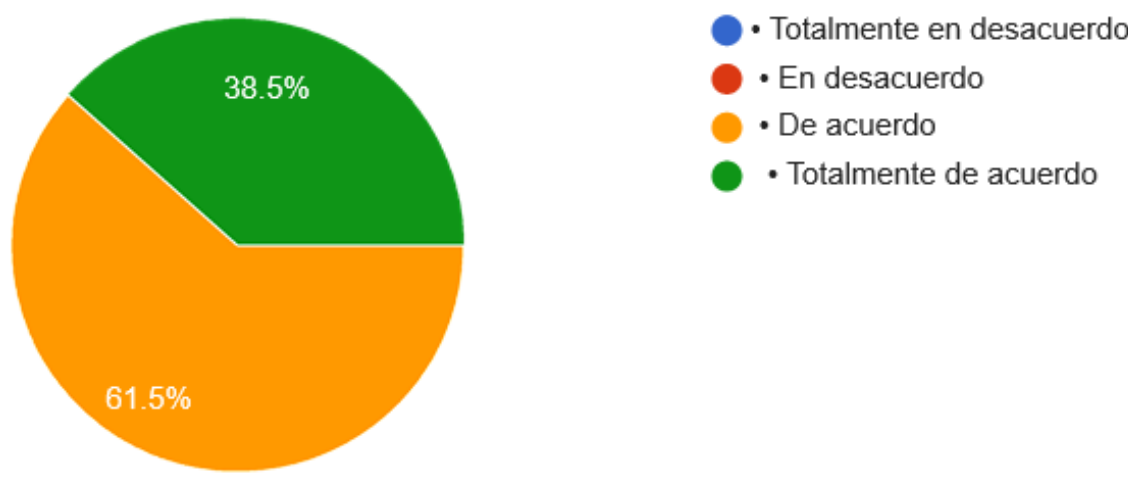

Grafico 1. Recursos tecnológicos para favorecer la enseñanza y aprendizaje a los estudiantes con discapacidad visual. 
El $61.5 \%$ de las personas encuestadas consideraron que el uso de los recursos tecnológicos favorecen el proceso de enseñanza y aprendizaje de los estudiantes con discapacidad visual, esto se debe a que con estos recursos se capta la atención del estudiante de mejor manera.

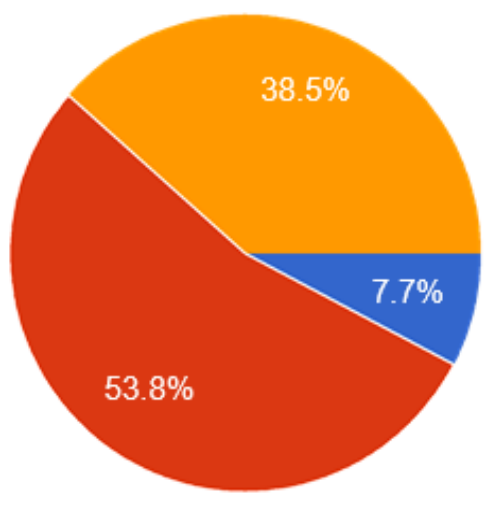

Seguidamente la pregunta 2 relacionada con cuales medidas consideraron los participantes del estudio, acerca de la existencia de los recursos digitales existentes y el fácil acceso que tenían los estudiantes con discapacidad visual (Gráfico 2).

Gráfico 2. Recursos digitales existentes de fácil acceso

El 53,8\% de los encuestados consideraron que es poco accesible los recursos digitales para estudiantes con discapacidad visual, esto puede deberse a la disponibilidad de los mismos, el costo que podría representar estos recursos específicos para estudiantes con discapacidad visual, y el factor económico, no todas las familias podrían tener la facilidad de acceder a estos tipos de recursos.

Continuando con la pregunta 3 valorar el nivel de conocimientos sobre el diseño de material digital accesible para estudiantes con discapacidad visual, a la población encuestada los resultados se muestran en el gráfico 3.

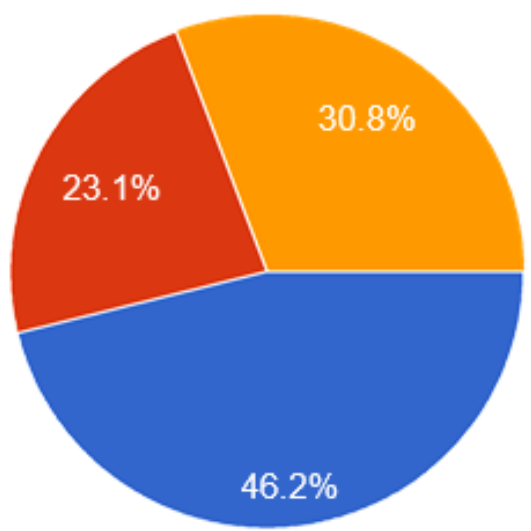

- Muy accesibles

- Poco accesibles

- Algo accesibles

- Nada accesibles 
El $46.2 \%$ reflejado en el gráfico 3 de los encuestados considera que su nivel de conocimientos es bajo para diseñar material para estudiantes con discapacidad visual, esto puede deberse a que para el desarrollo de este material se necesita conocimientos de programación, o no están muy familiarizados con la tecnología.

$\mathrm{Al}$ respecto, de si se conocen las pautas de accesibilidad para diseñar material digital accesible para estudiantes con discapacidad visual, respondieron lo siguiente en el gráfico 4.
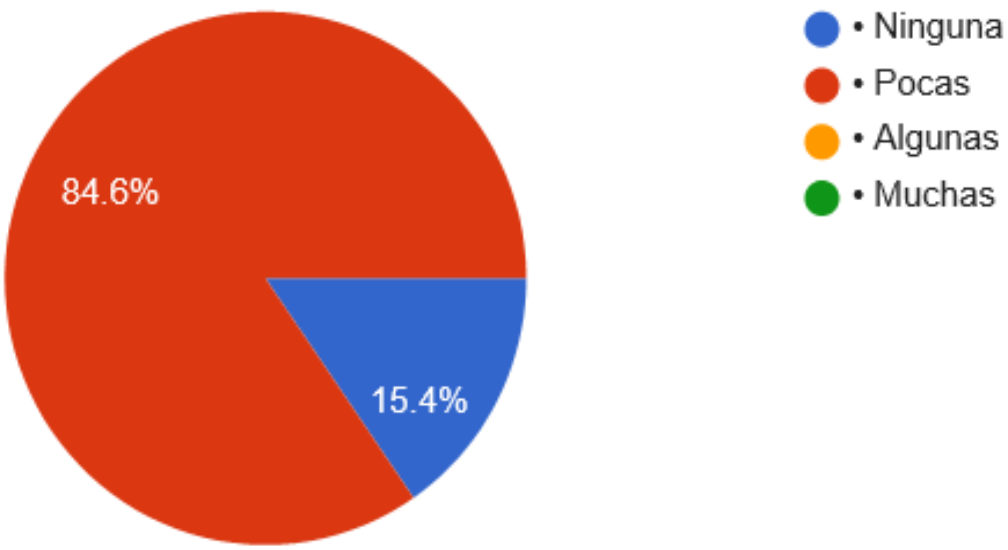

Gráfico 4. Pautas de accesibilidad para el diseño de un material digital.

El 84.6\% de los encuestados consideraron que son pocas las pautas de accesibilidad para diseñar material digital accesible para estudiantes con discapacidad visual, puede deberse a que no están del todo familiarizados con los entornos de desarrollo para este tipo de material digital.

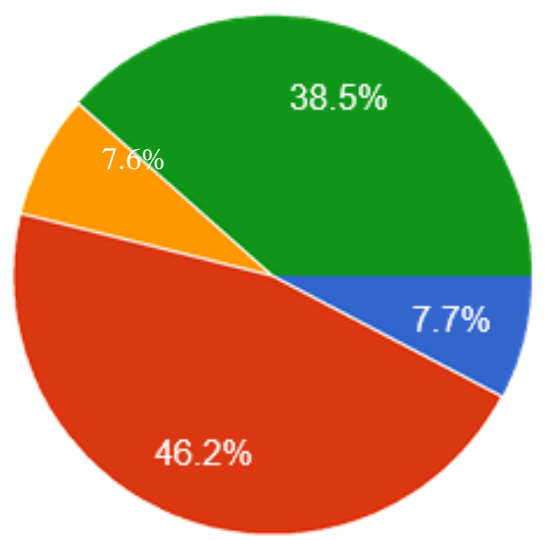

Gráfico 5. Aplicaciones y programas digitales sugeridos
Para que un recurso digital sea accesible debe ser trabajado inicialmente en una aplicación con ciertas pautas. Con relación a la interrogante ¿Desde cuál de los siguientes programas considera usted que se deben aplicar las mismas? Los resultados se muestran en el gráfico 5 .

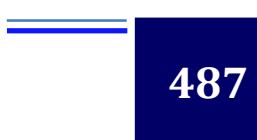


Se tuvo que el $46,2 \%$ de los encuestados consideraron que la plicacion Microsoft Word es el recurso digital, esto puede deberse a que están más familiarizados con esta herramienta digital. El 38.5\% de los encuestados considera que otro programa puede ser desde una plataforma de internet, pero se debe considerar el desarrollo de la plataforma.

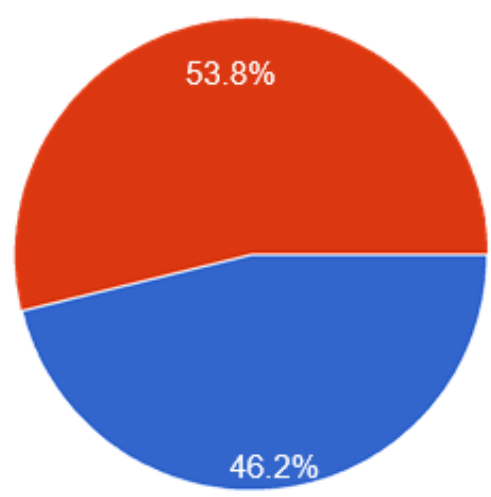

Una de las interrogantes que vale la pena señalar tuvo que ver con lo que se necesita para que un documento de Word sea accesible para un estudiante con discapacidad visual entorno así este debía tener imágenes a los que respondieron (Ver gráfico 6).

Gráfico 6. Necesidad de que un documento posea imágenes

En donde se destaca que, el 53.8\% consideró que la premisa es falsa, no se debe retirar las imágenes del documento, el documento debe igual contar con una descripción de la imagen.
Continuando con las interrogantes en la encuesta es presentada la relacionada con el recurso digital elaborado en el procesador de texto Word acerca de la accesibilidad para un estudiante con discapacidad visual (grafico 7).

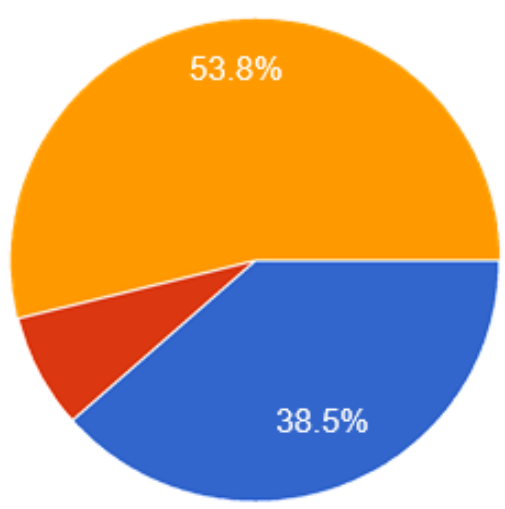

- Eliminar las tablas, los gráficos y enlaces de navegación

- Definitivamente no colocar ni tablas ni gráficos y enlaces de navegación

- Elaborar tablas, gráficos y enlaces de navegación accesibles

Gráfico 7. Recurso digital elaborado en el procesador de texto 
En el grafico 7 se muestra que se obtuvo que el $53.8 \%$ de los encuestados consideraron que la elaboración de tablas, gráficos y enlaces de navegación hace que Word se un recurso digital accesible para un estudiante con discapacidad visual.

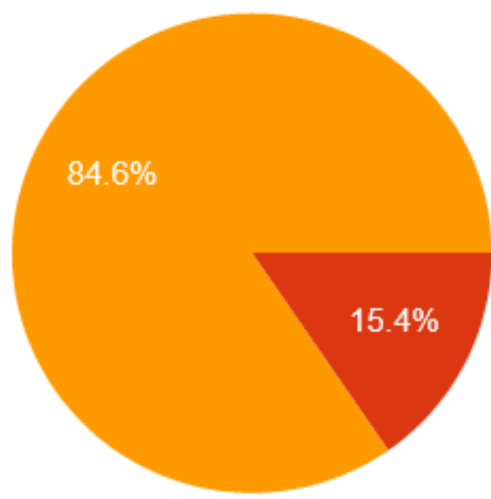

Gráfico 8. Programas y aplicaciones para transformar

En el gráfico anterior fue manifestado que el $84,6 \%$ de los encuestados prefieren usar un programa externo para poder transformar un documento de Word a PDF, solo el 15,4\% sabe que pueden realizarlo directamente desde el propio Word dejando entrever la importancia de esto en la mejora en la formación de los estudiantes con discapacidad visual.

Tras los resultados obtenidos de la aplicación de la encuesta, y como ya se analizaron previamente, los docentes carecían de los conocimientos para diseñar textos digitales accesibles para estudiantes con discapacidad visual, debido a esta necesidad surgió propuesta que me muestra a continuación, con la finalidad de contribuir a la mejora en los procesos de enseñanza $y$ aprendizaje a los estudiantes con discapacidad visual
Con relación a la interrogantes ¿Cuál de los siguientes programas o aplicaciones sirve para transformar un documento de Word a PDF?, en el gráfico se muestra las impresiones que fueron recogidas en el momento de la aplicación de la encuesta.

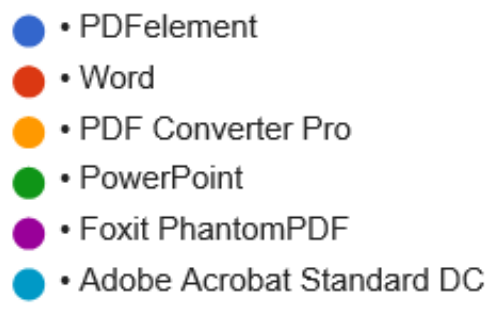

\section{Pautas y herramientas para el diseño de material digital accesible para estudiantes con discapacidad visual}

En el ámbito de la didáctica, todo docente se encuentra motivado a identificar, desarrollar y aplicar las mejores estrategias y recursos que actúen como mediadores en el proceso de enseñanza y aprendizaje para potencializar la adquisición de aprendizajes que resulten significativos para el estudiante, entre los que puede utilizar textos digitales en los principios del diseño universal o diseño para todos (Óscar, 2015).

Cuando el docente crea contenidos digitales en texto, debe tener en cuenta que entre los receptores se podrán encontrar personas con discapacidad visual que encuentren dificultad para acceder a toda la información, así como 
para aprovechar los aprendizajes en las mismas condiciones que todo el grupo, (Óscar, 2015).

En realidad, todos pueden presentar en algún momento de su vida y por diferentes motivos limitaciones y mermar temporalmente o condicionar la accesibilidad a contenidos digitales en texto; esto es evidente si presenta una discapacidad visual, sin embargo, aunque no todas la personas la presente, no quiere decir, que los se esté al margen de requerir alternativas diversas para aprovechar la información y recibir educación de este tipo.

Es necesario tomar en consideración que "Para elaborar un material educativo accesible, es importante hacer comprensible tres aspectos: su contenido, su estructura y su formato." (Óscar, 2015, p. 2). Por ejemplo, es posible que alguna vez sea necesario leer una noticia en medio de un entorno educativo, mientras son realizadas diferentes actividades; así también, está la incuestionable realidad de que con la edad se pierden algunas facultades, cuesta más acceder a la información, y se necesitará de diferentes apoyos que permitan mantener un buen estilo de vida.

Ya que el contenido expuesto debe ser redactado en lenguaje breve y sencillo, sin necesidad de utilizar estructuras gramaticales complejas. El mensaje que se desea transmitir debe ser concreto y directo, evitando en lo posible términos abstractos los cuales podrían resultar confusos. (Óscar, 2015, p. 3)

Siendo importante que el docente busque pautas prácticas en el que además de reforzar pautas y generar conocimientos relacionados con las ayudas técnicas de uso y aplicación sencilla como son por ejemplo los lectores de pantalla como él (JAWS Y NVDA) que utilizan habitualmente las personas con discapacidad visual, para poder "oír" la descripción de los contenidos visuales que no pueden ser vistos en la pantalla. (Sancho, 2009)
Se puede tomar en cuenta, para apoyar al docente a crear recursos accesibles utilizando algunos de los editores de contenidos digitales que componen las aplicaciones de Microsoft, a fin de crear documentos en procesadores de texto como Word accesibles, (Tafur, 2011).

Siendo así, al incorporar los textos digitales al proceso de enseñanza y aprendizaje se deberá considerar varias herramientas que permiten un amplio acceso a la información:

\section{Pautas para la creación de contenidos accesibles}

Los docentes en el proceso de enseñanza y aprendizaje deben considerar la implementación de materiales digitales de fácil acceso, hoy en día se podría destacar los textos digitales accesibles que son fácilmente transformables a diferentes formatos como mp3, para poder escucharlos, (Flores, 2012).

Así mismo, pueden ser utilizados por personas que tienen preferencias de accesibilidad o que acceden a los contenidos desde contextos especiales: Una persona ciega o que viaja en autobús podrá escuchar un texto en su teléfono móvil. Estas formas de uso serían impensables con los materiales docentes tradicionales,

Tomando en cuenta la utilidad en el ámbito educativo es que los materiales digitales de texto con los que se cuentan sean accesibles para leerlos con un lector de pantalla como el jaws o el nada.

Para que el docente pueda elaborar material educativo accesible, debe tomar en cuenta para hacerlos comprensible tres aspectos: su contenido, su estructura y su formato. Para lo cual se mencionan algunas características algunas características al elaborar un material de texto, en Word accesible. 
El texto tiene que ser claro como lo menciona Óscar, (2015). En cuanto al mensaje que se desea transmitir tiene que ser claro, coherentes y sencillo para lograr su compresión y aportar a su aprendizaje.

En cuanto al Idioma del documento, se sugiere que los estudiantes con discapacidad visual antes de utilizar su lector de pantalla deben configurar el idioma de su computador para tener fluidez y evitar una pronunciación errónea y si existen palabras en otro idioma configurarlas independientemente.

Para lo cual, se sugiere seguir algunas recomendaciones de la Organización Nacional de Ciegos Españoles (ONCE) (Islas, 2017).

Enlistando las siguientes pautas para tomar en cuenta al momento de diseñar material digital en Word accesible. Tipos de letra, se puede utilizar Verdana o Arial que favorece a la baja visión. Tamaño de fuente, se debe utilizar fuentes de 12 y 14 puntos si existe residuo visual.

Se recomienda utilizar las mayúsculas en palabras cortas. En el caso de los títulos el texto se lee con mayor facilidad cuando está escrito en tipo oración. El espacio entre una línea y la siguiente será aproximadamente un $25-30 \%$ del tamaño del punto. El color del papel y de la tinta deberá ser blanco o amarillo y la tinta aprovechado la agudeza visual. En cuanto posición del texto, justificación, se recomienda que no estén escritos en horizontal. Para justificar los textos se tendrá que hacer al a izquierda, pues esto ayuda a encontrar el principio del renglón. (Tafur, 2011).

Para poder complementar el diseño de material accesible, también hay que tomar en cuenta las siguientes pautas: En ampliador de pantalla; esquematización de contenidos, se realiza en la pestaña "Inicio", sección "Estilos", se establece el formato deseado en el texto del documento.
Estilos para texto normal y títulos de nivel 1,2 y 3 , se selecciona el estilo del texto presionando simultáneamente las teclas de acuerdo a las combinaciones, por ejemplo Estilo normal: "Ctrl + Mayús + A". (Óscar, 2015).

Espacio entre líneas y párrafos para la creación de espaciado entre líneas y párrafos; con el uso de ratón: Pestaña "Inicio", sección "Párrafo" opción "Espaciado entre líneas y párrafos” (Óscar, 2015).

Saltos de sección y de página, para la creación de listas numeradas o viñetas: hay que considerar como opción el uso del teclado: Escribir un asterisco para empezar una lista con viñetas o el número 1 para iniciar una lista numerada $\mathrm{y}$, a continuación, presionar las teclas "Barra espaciadora" o "Tab", automáticamente se genera una lista. (Óscar, 2015).

Creación de listas, columnas y tablas, el texto en más de una columna, se recomienda utilizar la herramienta adecuada para ello, evitando el uso del tabulador para separar texto o simularlo mediante tablas. (Óscar, 2015).

Al crear columnas de texto. Instrucciones para la creación de columnas de texto: Mediante el uso de teclado: Presionar "Alt $+\mathrm{C}$ " para acceder a la pestaña "Diseño de página", presionar la tecla "J" para seleccionar el menú "Columnas". Planteado por (Óscar, 2015).

Para las tablas de contenido: realizar con el teclado: Presionar tecla "Alt $+\mathrm{S}$ " para activar la pestaña "Referencias", presionar tecla "T" para activar el menú "Tabla de contenido", con las flechas direccionales arriba-abajo para seleccionar cualquiera de las dos opciones de presentación de contenidos.

Descripción de imagen. Como en el caso de las imágenes, las gráficas deben ser accesibles para todos los usuarios en general tomando en cuenta las siguientes Instrucciones para asignar texto alternativo a una imagen: 
- Paso 1: Posicionarse en la imagen, seleccionar "Formato de imagen.

- Paso 2: En la ventana "Formato de imagen" seleccionar la opción "Texto alternativo" y escribir una descripción de la información que proporciona la imagen. Como menciona (Óscar, 2015).

Cuando se utilizan enlaces de navegación se debe realizar los siguientes pasos

- Paso 1: Seleccionar en la pestaña "Insertar", la opción "Hipervínculo".

- Paso 2: Introducir el texto del enlace, la dirección y seleccionar la opción "Info. En pantalla", para incluir la descripción a dónde lleva ese enlace para que los usuarios tengan más orientación.

Finalmente, acceder a las herramientas de validación de accesibilidad y por ultimo convertir un documento en PDF, (Óscar, 2015).

Para que el estudiante con discapacidad visual pueda acceder con su lector de pantalla; las indicaciones deben tomar en cuenta la participación del docente como algo esencial para mejorar la participación de un estudiante con discapacidad visual, en los procesos educativos, logrando su independencia en las actividades académicas, dentro del aula, y así poder acceder al a información en su casa con autonomía. (Tafur, 2011).

\section{Resultados obtenidos}

Con la finalidad de evaluar la propuesta presentada sobre el diseño de textos digitales accesibles para estudiantes con discapacidad visual, se realizó una evaluación por parte de expertos en el campo de la educación de personas con discapacidad visual, con la finalidad de determinar el nivel de éxito en la aplicación de esta propuesta. El juicio de expertos es un método de validación útil para verificar la fiabilidad de una investigación que se define como "una opinión informada de personas con trayectoria en el tema, que son reconocidas por otros como expertos cualificados en éste, y que pueden dar información, evidencia, juicios y valoraciones" (Escobar-Pérez y Cuervo-Martínez, 2008, p. 29).

Continuando con el análisis se aplicó una encuesta a dieciséis (16) expertos, dicho instrumento está dividido en 4 categorías que son las siguientes: claridad, pertinencia, coherencia y relevancia. Cada una de estas categorías cuenta con una serie de preguntas que fueron valoradas con una escala de 1 a 5 , donde fueron considerado los siguientes equivalentes: 1 totalmente en desacuerdo; 2 en desacuerdo; 3 de acuerdo; 4 muy de acuerdo, totalmente de acuerdo.

En el gráfico 9, es presentado los resultados estadísticos obtenidos de la aplicación de la encuesta al grupo de expertos acerca de la evaluación de la propuesta de diseño de textos digitales accesibles para estudiantes con discapacidad visual. 


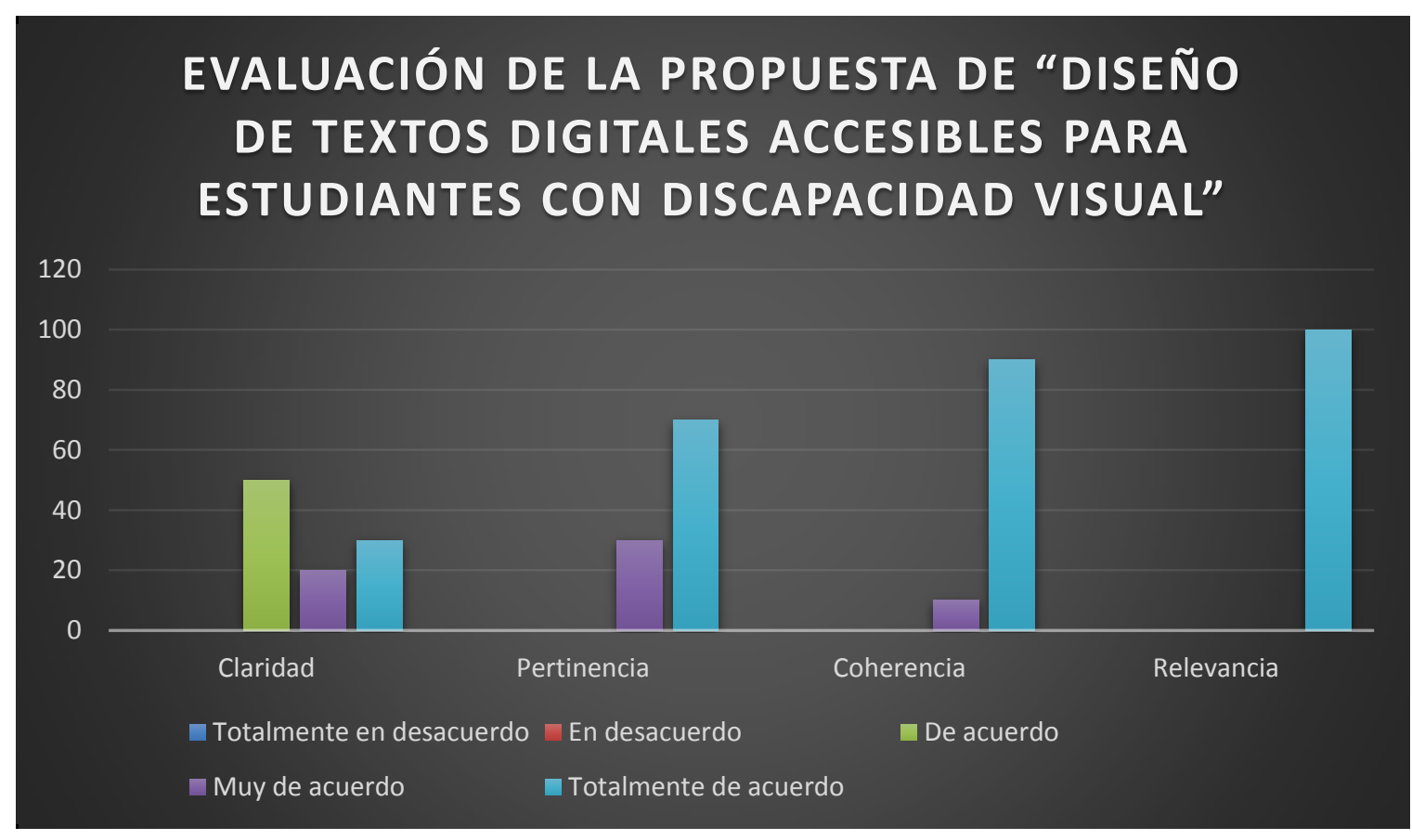

Gráfico 9. Resultados de la evaluación de la propuesta

\section{Análisis de los resultados}

La propuesta presentada sobre el diseño de textos digitales accesibles para estudiantes con discapacidad visual, en su mayoría fue valorada positivamente, en el análisis de los resultados obtenidos de acuerdo con las categorías propuestas en la encuesta de validación son detallas seguidamente.

Con respecto a la primera categoría presentada sobre claridad, se obtuvo que el $50 \%$ de los expertos están de acuerdo, otro $20 \%$ muy de acuerdo y el 30\% totalmente de acuerdo. La dimensión correspondiente a la claridad de la propuesta fue la que menor puntuación obtuvo, esto debido a que la mayoría de expertos consideran que no está presentada de manera secuencial, para que quien la revise pueda seguir los pasos que exige su aplicación. Entre las recomendaciones obtenidas, fue destacada la redacción de la propuesta presentando una secuencia clara, inclusive con numeración, con la finalidad de que el diseño del texto accesible se lo haga siguiendo un determinado orden.

Por otro lado, en referencia a la segunda categoría de la encuesta, correspondiente al grado de pertinencia de la propuesta, los expertos en general respondieron que consideran que la misma presenta una estrecha relación con el objetivo que persigue, el cual es el de mejorar el proceso educativo de estudiantes con discapacidad visual. Es así que el 30\% de los expertos respondieron estar muy de acuerdo, mientras que el $70 \%$ manifiesta estar totalmente de acuerdo con el cumplimiento del objetivo planteado, lo cual permite deducir que efectivamente la propuesta mejoraría el proceso de enseñanza y aprendizaje de los educandos con discapacidad visual.

Con respecto a la categoría de coherencia existe un $10 \%$ de los docentes que están muy de acuerdo con la propuesta, ya que guarda relación lógica con la destreza que se busca

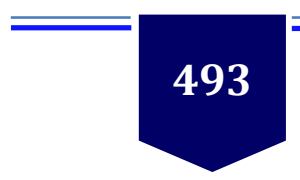


potenciar y el $90 \%$ totalmente de acuerdo con la propuesta, manifestando que guarda relación lógica con la destreza que se busca potenciar. Aplicando las pautas para crear textos accesibles para estudiantes con discapacidad visual, para usarlos con un lector de pantalla

Finalmente, en relación a la categoría de relevancia, la totalidad\% del personal de la institución están totalmente de acuerdo con la propuesta que permite que la población objetiva mejore su calidad de vida o ejerza un derecho humano, contribuyendo al proceso de inclusión educativa, mejorando su autoestima y al acceso a la información aportando la participación en los procesos de aprendizaje entre pares y con el docente.

\section{Discusión}

Según los resultados previamente señalados el estudio se centró en las necesidades educativas digitales del colectivo con discapacidad visual, puesto que los avances tecnológicos han facilitado en gran medida el acceso a la información, convirtiéndose en un apoyo importante en el proceso educativo, laboral, social, cultural entre otros, los estudiantes con necesidades visuales han logrado acceder a la información, tomando en consideración que sin estos recursos se les dificulta su labor para obtener el aprendizaje.

Es importante indicar que en concordancia con el actual modelo social de la discapacidad, así como también con el enfoque de la inclusión educativa, La discapacidad visual puede ser definida como:

La dificultad que presentan algunas personas para participar en actividades propias de la vida cotidiana, que surge como consecuencia de la interacción entre una dificultad específica relacionada con una disminución o pérdida de las funciones visuales y las barreras presentes en el contexto en el que se desenvuelve la persona (Ministerio de Educación de Chile, 2014). Por lo tanto, la discapacidad visual es una condición que engloba diferentes tipos de dificultades visuales. Supone una pérdida en el sistema perceptivo y de acceso a la información del entorno, ya que el $80 \%$ de la información que llega del exterior es de tipo visual. Las personas con discapacidad visual presentan diferente grado de visión, por lo que los apoyos que cada uno requieren estar determinadas por el tipo de déficit visual y por su funcionalidad visual. Cabe indicar que La discapacidad visual es un término genérico, el cual engloba tanto a la ceguera como a la baja visión, existiendo diferencias significativas entre estas 2 condiciones, tal como lo manifiesta Mon (como se citó en Aquino, García e Izquierdo, 2012) la baja visión hace referencia a deficiencias a nivel de la agudeza y/o del campo visual, es decir, si bien existen limitaciones en el funcionamiento visual, las personas con baja visión, dependiendo del grado, tendrían posibilidades de usar su remanente visual para movilizarse o realizar diferentes actividades de la vida cotidiana. Por otro lado, según este mismo autor, la ceguera hace referencia a la pérdida total de la visión o a un remanente visual mínimo, en cualquiera de estas dos situaciones los recursos tecnológicos constituyen una herramienta facilitadora para la adquisición de conocimientos.

Resulta necesario conocer las características generales de la discapacidad visual, así como también los diferentes apoyos indispensables para la inclusión educativa de los estudiantes que presentan esta condición. En el ámbito educativo, Este tipo de limitación se caracteriza por sus dificultades para acceder a la información impresa y digital cuando la misma no es diseñada considerando ciertas pautas de accesibilidad. 
Por todo ello, surge la necesidad de brindar una propuesta para diseñar textos digitales accesibles para estudiantes con discapacidad visual.
Cabe indicar que en el Ecuador el 11,60\% de personas presenta algún tipo de discapacidad visual, tal como se muestra en el gráfico a continuación:

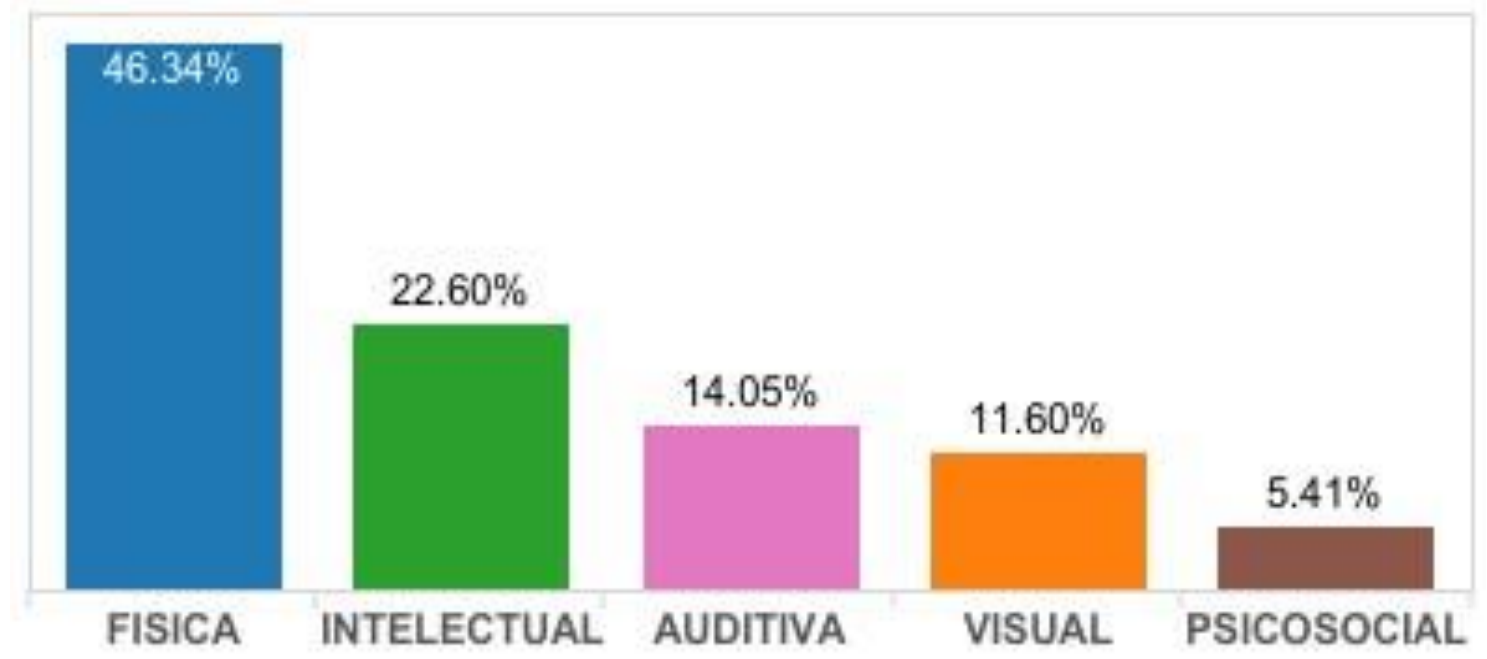

Figura 1. Tipos de discapacidad. Tomado del Consejo Nacional para la Igualdad de Discapacidades del Ecuador a junio de 2020.

Tal como se observa, la discapacidad visual ocupa el cuarto lugar en orden porcentual en Ecuador, lo cual como sociedad, y aún más como sistema educativo inclusivo, compromete al sistema educativo con la mejora dentro del proceso enseñanza $\mathrm{y}$ aprendizaje de este colectivo.

En la Constitución de la República del Ecuador (2008) el Art. 26 establece que la educación es un derecho de las personas a lo largo de su vida y un deber ineludible e inexcusable del Estado. Constituye un área prioritaria de la política pública y de la inversión estatal, garantía de la igualdad e inclusión social y condición indispensable para el buen vivir. Seguidamente en la línea de inversión estatal y garantía de una educación inclusiva, Ecuador en su ley Orgánica de Educación Intercultural (LOEI), En el Título III, capítulo primero referente a los objetivos del sistema nacional de educación, específicamente en el artículo 19, establece lo siguiente: "El Estado en todos sus niveles de gobierno y en ejercicio concurrente de la gestión de la educación, planificará, organizará, proveerá y optimizará los servicios educativos considerando criterios técnicos, pedagógicos, tecnológicos, culturales, lingüísticos, de compensación de inequidades y territoriales" (LOEI, 2011, p. 18).Tal como se estipula en este artículo de la LOEI, el estado deberá proveer de recursos tecnológicos, aspecto clave para que la educación vaya en línea con los actuales avances científicos y tecnológicos que se están dando a nivel mundial.

Entonces, la legislación ecuatoriana contempla tanto a nivel de su carta magna, como en muchas de sus leyes orgánicas 
artículos referentes a la igualdad e inclusión social, por lo que a nivel educativo constantemente se escucha y se lee los discursos sobre la "educación inclusiva", frase en muchas ocasiones carente de un significado en la praxis. Lamentablemente, aún se puede observar que los docentes carecen de conocimientos y herramientas para responder a la diversidad, tanto de estilos, como de ritmos de aprendizaje, por lo tanto, aún no se puede manifestar que existan verdaderos procesos educativos inclusivos, tal como lo manifiesta López (2011):

mientras haya un alumno o una alumna en una clase que no haya logrado su dignidad, es decir, que no sea respetado como es, ni participe en la construcción del conocimiento con los demás ni conviva en igualdad de condiciones que el resto de compañeros y compañeras, no habremos alcanzado la educación inclusiva (López, 2011, p. 40).

Por lo tanto, la inclusión requiere de un respeto por la diversidad, así como también, conseguir una plena participación de todos los educandos independientemente de sus condiciones particulares. En consecuencia, para lograr que esa participación sea una realidad en el ámbito educativo, se necesita de materiales didácticos accesibles para todos los educandos, específicamente en el caso de la población estudiantil con discapacidad visual requiere contar con recursos digitales adecuados a sus necesidades educativas.

Por todo ello, la educación inclusiva tiene necesariamente que realizar la detección de aquellas barreras del contexto, para a partir de ello, emprender acciones que permita adecuar el entorno, o simplemente buscar alternativas que permitan disminuir o incluso eliminar aquellos obstáculos en la educación de nuestros estudiantes. Ainscow, 2004 (como se citó en López, 2011) el concepto de "barreras" hace referencia a cómo, por ejemplo, la falta de recursos o de experiencia o la existencia de un programa, de métodos de enseñanza y de actitudes inadecuadas pueden limitar la presencia, la participación y el aprendizaje de determinados alumnos y alumnas. Por ende, tanto la falta de existencia de recursos digitales accesibles para estudiantes con discapacidad visual, así como la falta de conocimiento por parte de los docentes al momento de diseñar este tipo de material, constituye una barrera para el logro de una verdadera educación inclusiva de este colectivo.

En consecuencia, el estudio se centró en el diseño de recursos digitales, los cuales según Toledo (2012) presentan ciertas características que los diferencia de los recursos didácticos tradicionales; como por ejemplo estos pueden ser utilizados por los estudiantes en cualquier momento y en cualquier espacio, así mismo pueden ser presentados en diferentes formatos como textuales, icónicos, audio-visuales y gráficos. Los recursos digitales textuales, constituyen la principal fuente de información requerida por los estudiantes con discapacidad visual. Los recursos educativos digitales son un "conjunto de materiales digitalizados, producidos con el objetivo de facilitar el desarrollo de actividades de aprendizaje. Así, su objetivo es favorecer la transmisión de conocimientos, la adquisición de habilidades e incluso el fomento de determinados valores" Zapata (como se citó en Pérez, 2017, p. 9), basado en la definición planteada, se puede deducir que la finalidad de la producción de recursos digitales es la de facilitar el proceso de aprendizaje de los educandos, sin embargo, cuando estos materiales no están elaborados para responder a las necesidades 
educativas de la población estudiantil con discapacidad visual constituyen una barrera al proceso de inclusión de este colectivo.

Por ende, para que un recurso digital, en este caso los textos, sean materiales facilitadores del proceso de enseñanza y aprendizaje, es necesario que cumplan con ciertos parámetros de accesibilidad. Entendiéndose la misma como "una cualidad, un requisito básico que debieran cumplir los espacios, ámbitos, servicios, bienes, etc." (Mareño, Masuero y Beltramone (como se citó en Escribano y Martínez, 2013). De igual manera en el ámbito educativo se debe considerar que los recursos didácticos que vayan a ser utilizados cumplan con ciertas condiciones que permitan que los mismos, sean utilizados por los educandos de forma autónoma, confortable y equitativa.

\section{CONCLUSIONES}

Con respecto al primer objetivo planteado, sobre el análisis teórico en relación a la inclusión educativa y la accesibilidad digital para estudiantes con discapacidad visual, se determinó que todo proceso inclusivo requiere que sea el entorno el que se adapte a las características de los educandos, y que de manera específica en el caso del colectivo con esta limitación, la tecnología, y de manera particular los recursos digitales constituyen un valioso recurso para facilitar el acceso a la información por medio de los lectores de pantalla.

Con respecto al nivel de conocimientos del personal docente y técnico de la institución educativa sobre el diseño de textos accesibles para estudiantes con discapacidad visual, se puede decir que la mayoría del personal considera que los recursos tecnológicos son sumamente importantes en el proceso de enseñanza-aprendizaje, sin embargo el conocimiento sobre accesibilidad digital de los profesionales y facilitadores es limitado, esto resulta contraproducente al momento de generar estrategias dentro del aula, ya que aplicar de forma errónea un recurso es una barrera y no una ayuda especializada.

La encuesta permitió el manifiesto y el menester para que los docentes conozcan el uso correcto, tanto de plataformas y software de uso cotidiano como WORD y PDF para facilitar lectores de pantalla, así como también los conocimientos básicos sobre textos digitales accesibles cuando se trabaja con NNA con discapacidad visual.

Con respecto a la valoración de la propuesta, tras la aplicación de la técnica de criterios de expertos, se determinó que la misma es pertinente, coherente y sumamente relevante para conseguir la mejora del proceso educativo del colectivo con discapacidad visual. Así mismo, resulta necesario indicar que de acuerdo con las sugerencias de los expertos, el aspecto que se debería mejorar es en relación a la claridad de la propuesta, puesto que consideran que sería mejor redactar la propuesta siguiendo un orden lógico.

\section{REFERENCIAS}

Albert, M. (2007). La investigación educativa. Madrid, España: McGraw-Hill, Obtenido de: https://bit.ly/32m9ETN

Aquino, S., García, V., e Izquierdo, J. (2012). La inclusión educativa de ciegos y baja visión en el nivel superior. Un estudio de caso. Sinéctica, 39. Recuperado de http://www.scielo.org.mx/pdf/sine/n39/ n39a7.pdf

Asamblea Nacional del Ecuador. (2008). Constitución política de la república del ecuador

Asamblea Nacional del Ecuador. (2011). Ley Orgánica de Educación Intercultural. 
Consejo Nacional para la Igualdad de Discapacidades del Ecuador 2020. Tipos de discapacidad. Recuperado de https://url2.cl/qKty7

Escribano, G. A., y Martínez, C. A. (2013). Inclusión educativa y profesorado inclusivo: Aprender juntos para aprender a vivir juntos. Madrid, España

Flores, V. (2012). Producción de materiales didácticos para estudiantes con discapacidad visual. Buenos Aires: Daniela Rovatti. Recuperado de : https://www.ucm.es/data/cont/media/w ww/pag-

83274/Manual\%20Pr\%C3\%A1ctico\%20p ara\%20Hacer\%20Textos\%20Accesibles\% 20para\%20Estudiantes\%20con\%20Diver sidad\%20Funcional.pdf

Islas, B. (2017). PAUTAS PARA LA CREACIÓN DE DOCUMENTOS DE TEXTO DIGITALES ACCESIBLES PARA PERSONAS CON CEGUERA. Barcelona: ONCE. Recuperado de

https://www.foal.es/sites/default/files/d ocs/17_MDVisual_web.pdf

López, M. (2011). Barreras que impiden la escuela inclusiva y algunas estrategias para construir una escuela sin exclusiones. Innovación Educativa,

Ministerio de Educación de Chile. (2014). Guía de Apoyo Técnico Pedagógico: Necesidades Educativas Especiales asociadas a la Discapacidad Visual en el Nivel de Educación Parvularia. Recuperado de http://especial.mineduc.cl/wpcontent/uploads/sites/31/2016/08/Guia Visual.pdf

Óscar, Á. F. (2015). Guía para crear contenidos digitales accesibles. Alcalá de Henares: Hilera González, José Ramón Campo Montalvo, Elena. Recuperado de : https://educacion.once.es/cres/crebarcelona/recursos/pautas-para-lacreacion-de-documentos-de-texto- digitales-accesibles-para-personas-conceguera

Pérez, I. (2017). Creación de recursos educativos digitales: Reflexiones sobre innovación educativa con TIC. Revista Internacional de sociología de la educación, vol. 6, núm. 2, pp. 243-268. Barcelona, España. Recuperado de https://www.redalyc.org/pdf/3171/3171 51451004.pdf

Salinas, J. (2014). El papel de las TICS en el sistema educativo. Universidad de las Illes Balears. Recuperado de https://www.researchgate.net/publicatio n/232242301_El_papel_de_las_TIC_en_el_s istema_educativo

Sancho, D. (2009). Manual de Teclas y Comandos de JAWS Para personas con disCapacidad Visual Programa Microsoft Windows XP-Vista Programa Microsoft Word 2007 Programa Microsoft Excel 2007 Programa Microsoft Power Point 2007. San José - Costa Rica: CMTC. Recuperado de: http://www.esvial.org/wpcontent/files/ESVIAL.LibroDigital_es_201 5.pdf

Tafur, A. (2011). MANUAL PRÁCTICO PARA HACER TEXTOS ACCESIBLES PARA ESTUDIANTES CON DIVERSIDAD FUNCIONAL. Madrid: Universidad Complutense de Madrid. Recuperado de : https://profesordiegosancho.webs.com/C MTC/04\%20-

\%20Comandos\%20JAWS\%200FFICE\%20 2007.pdf

Toledo, G. A. (2012). Accesibilidad digital para usuarios con limitaciones visuales (Doctoral dissertation, Universidad Nacional de La Plata). Recuperado de http://sedici.unlp.edu.ar/bitstream/handl e/10915/24871/Documento_completo_. \%20Toledo\%20Gabriela\%20UNLP112012 modif.pdf?sequence=1\&isAllowed $=y$ 